\title{
中医药防治新型冠状病毒肺炎的优势与挑战
}

黄璐琦

中国中医科学院中国中医药循证医学中心, 北京 100700

E-mail: huangluqi01@126.com

\section{Advantages and challenges of Traditional Chinese Medicine in the prevention and treatment of COVID-19}

\section{Luqi Huang}

China Center for Evidence-based Medicine of TCM, China Academy of Chinese Medical Sciences, Beijing 100700, China E-mail: huangluqi01@126.com

doi: 10.1360/TB-2021-0837

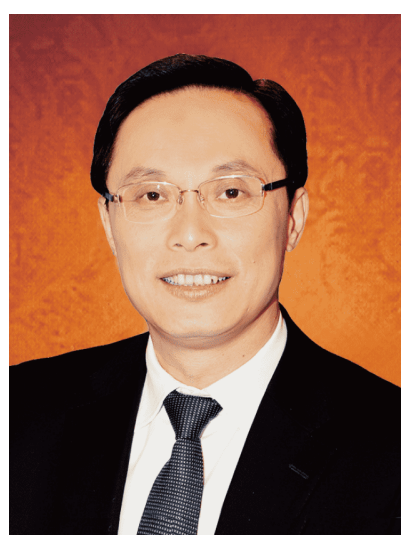

\section{黄路绮}

中国工程院院士，国家中医药管理局副 局长，中国科学技术协会副主席，中国中 医科学院院长. 获国家杰出青年科学基 金资助。曾任首批国家中医援鄂医疗队 领队、国家中医药防治新型冠状病毒肺 炎专家组组长、科研攻关组组长, 主要 从事分子生药学、中药资源学的研究.
新型冠状病毒肺炎(COVID-19，简称“新冠肺炎”)在全球范围内不断 蔓延, 严重威胁人类健康, 对全球政治、经济产生深远影响. 经过全球科 学家的不断努力, 疫苗及时问世。但随着新冠肺炎变种病毒的不断出现, 人类对于新冠肺炎的整体防控仍然任重道远. 中医药是全球新冠肺炎疫 情防控“中国方案”的优势与亮点. 2020年至今中国抗击新冠肺炎疫情过 程中，中医药深度介入预防、治疗、康复的全过程，与西医优势互补、相 互协作, 为全面控制新冠肺炎疫情作出重要贡献, 发挥了独特作用. 基于 中医疫病理论与古代经典名方, 在中医药防治新冠肺炎的临床实践中, 篎 选出金花清感颗粒、连花清瘟胶囊、血必净注射液和清肺排毒汤、化湿 败毒方、宣肺败毒方等有明显临床疗效的方药——三药三方”。“三药三 方”被纳入国家新冠肺炎诊疗指南, 得到广泛应用, 取得较好的疗效. 在中 医药临床救治的同时, 积极开展临床研究, 用科学的方法阐明中医药防治 新冠肺炎的有效性和安全性, 发表了一系列的高水平临床研究成果, 如首 个也是唯- - 项在武汉市金银潭医院规范、完整实施并报告的中医药治 疗新冠肺炎随机对照试验(randomized controlled trial, RCT)表明, 化湿败 毒颗粒对新冠肺炎住院患者临床症状(发热、咳嗽、乏力、胸间)和肺部 炎症有明显的改善作用, 且安全性良好 ${ }^{[1]}$. 这为中医药防治新冠肺炎的临 床应用提供了高质量的循证证据，体现了中医药在防治新发急性传染病 中的巨大临床价值.

为科学评价新冠肺炎疫情期间开展的中医药相关研究, 立足前沿、 总结经验、展望未来, 我们邀请相关学者撰写文章组成本专题. 香港浸 会大学市兆祥教授对中医药防治新冠肺炎临床研究的总体状况进行探讨, 以期为中医药在未来重大/突发/新发疾病防治中，提供更强的证据基础 ${ }^{[2]}$. 澳门科技大学中医药学院徐友华教授团队系统总结了中医药对新冠肺炎 诱导的炎症反应的干预作用及机制，同时分析了中医药对新冠肺炎不同 
病情患者的应用差异性 ${ }^{[3]}$. 中国中医科学院团队对2020年1月 2021年3月期间发表的中医药干预新冠肺炎临床研 究文献开展文献计量学分析, 通过不同维度的系统整理, 对其整体现况、实施内容及对新冠肺炎的中医药治疗效 果进行定性与定量分析, 同时开展解读与思考 ${ }^{[4]}$. 以上文章对理性认知中医药防治新冠肺炎的研究现状具有重要 意义.

中医药临床疗效评价与成果转化是支持中医药发展的重要研究领域, 在这次防控新冠肺炎疫情过程中, 中医 药参与面之广、参与度之深、受关注程度之高, 是新中国成立以来前所未有的. 值得欣喜的是, 国家药品监督管理 局2021年3月2日正式批准中医药“三方”——清肺排毒颗粒、化湿败毒颗粒、宣肺败毒颗粒上市，将中医药科技原 创优势转化为重大成果. 面对当前世界文化的多元化与医疗学术格局, 机遇与挑战并存, 应积极推动解决中医药当 前临床疗效评价方面存在的瓶颈问题. 立足国际高度, 以合理客观评价中医药临床疗效与安全性为根本, 提升中医 药临床证据支持决策的能力, 促进中医药产业发展, 支持中医药走向世界 ${ }^{[5]}$.

\section{参考文献}

1 Liu J, Yang W, Liu Y, et al. Combination of Hua Shi Bai Du Granule (Q-14) and standard care in the treatment of patients with coronavirus disease 2019 (COVID-19): A single-center, open-label, randomized controlled trial. Phytomedicine, 2021, doi: 10.1016/j.phymed.2021.153671

2 Bian Z X. Improving the quality of clinical research for the prevention and treatment of COVID-19 with Traditional Chinese Medicine. Chin Sci Bull, 2021, 66: 3372-3376 [市兆祥. 中医药防治新冠肺炎临床证据质量的提升. 科学通报, 2021, 66: 3372-3376]

3 Li Y T, Wang Z, Lin J, et al. Progress on the role of Traditional Chinese Medicine in inflammatory injury of COVID-19. Chin Sci Bull, 2021, 66: 3377-3384 [李云制, 王吉, 林静, 等. 中医药在新型冠状病毒肺炎炎症损伤中的作用研究进展. 科学通报, 2021, 66: 3377-3384]

4 Li Y W, Liu Y, Lü C, et al. Clinical trials for Traditional Chinese Medicine against coronavirus disease 2019 (COVID-19): Interpretation based on bibliometric analysis. Chin Sci Bull, 2021, 66: 3385-3394 [李逸雯, 刘玥, 吕诚, 等. 新型冠状病毒肺炎的中医药临床研究: 基于文献计量学分 析的解读与思考. 科学通报, 2021, 66: 3385-3394]

5 Wang Y Y, Huang L Q. Taking a broad and long-term view to establish China Center for Evidence Based Traditional Chinese Medicine (CCEBTCM). Chin J Evid-Based Med, 2019, 19: 1131-1137 [王永炎, 黄璐琦. 立足高远, 建设中国中医药循证医学中心. 中国循证医学杂志, 2019, 19: 1131-1137] 